doi: ISSN 0103-5150

Fisioter. Mov., Curitiba, v. 26, n. 4, p. 921-932, set./dez. 2013

Licenciado sob uma Licença Creative Commons

\title{
Tratamento fisioterapêutico para epicondilite lateral: uma revisão sistemática
}

\section{Physiotherapy treatment for tennis elbow: a systematic review}

\author{
Matheus Oliveira de Almeida ${ }^{[\mathrm{a}]}$, Bruno Tirotti Saragiotto ${ }^{[\mathrm{a}]}$, Tiê Parma Yamato ${ }^{[\mathrm{a}]}$, \\ Raphael Lobão Pereira ${ }^{[a]}$, Alexandre Dias Lopes ${ }^{[\mathrm{b}]}$
}

[a] Mestres em Fisioterapia pela Universidade Cidade de São Paulo (UNICID), membros do São Paulo Running Injury Group (SPRunIG), São Paulo, SP - Brasil, e-mails: matheus.almeida@sprunig.net, bruno.saragiotto@sprunig.net, tie.yamato@sprunig.net, raphalobao@gmail.com

[b] Professor do Programa de Mestrado em Fisioterapia da Universidade Cidade de São Paulo (UNICID), membro do São Paulo Running Injury Group (SPRunIG), São Paulo, SP, Brasil, e-mail: alexandre.lopes@sprunig.net

\section{Resumo}

Introdução: Embora o tratamento conservador ainda seja a melhor conduta inicial para a epicondilite lateral do cotovelo, há pouca evidência científica de que o tratamento fisioterapêutico altere o curso natural da doença e seja efetiva no tratamento dessa patologia. Objetivo: Avaliar a efetividade e a segurança das diversas intervenções fisioterapêuticas utilizadas no tratamento conservador da epicondilite lateral. Materiais e métodos: Foram incluídos apenas ensaios clínicos aleatorizados e quase aleatorizados que utilizaram pelo menos uma modalidade fisioterapêutica como uma das intervenções. Foi realizada uma busca nas bases de dados eletrônicos MEDLINE; Embase; LILACS e SciELO até dezembro de 2010. Não houve restrição do período de publicação dos artigos. Com o objetivo de aumentar a sensibilidade e a precisão, a estratégia de busca utilizada foi adaptada para cada base de dados. Foi utilizada a pontuação da escala PEDro para avaliação da qualidade metodológica dos ensaios clínicos aleatorizados. Resultados: 26 artigos foram incluídos na revisão sistemática e a avaliação da maioria desses artigos apresentou qualidade metodológica satisfatória (6,4 pontos). Em relação aos aspectos mensurados, todos os artigos avaliaram a dor, 18 (69\%) examinaram a força de preensão e $11(42 \%)$ a função dos pacientes. Quanto à duração do acompanhamento dos pacientes apenas nove (35\%) artigos realizaram acompanhamento a longo prazo. Conclusão: A melhor opção para o tratamento da epicondilite lateral parece ser a combinação de modalidades terapêuticas, o que condiz com a realidade clínica do 
fisioterapeuta. Porém, mais ensaios clínicos com boa qualidade metodológica são necessários para determinar a efetividade da maioria das modalidades terapêuticas encontradas.

Palavras-chave: Cotovelo de tenista. Reabilitação. Fisioterapia.

\section{Abstract}

Introduction: Although conservative treatment is still the best initial management for lateral epicondylitis, there is little scientific evidence that physical therapy change the natural history of disease and is effective for treating this condition. Objective: evaluate the effectiveness and safety of different physical therapy interventions used in the conservative treatment of lateral epicondylitis. Materials and methods: We included only randomized controlled trials and quasi-randomized studies that used at least one modality of physical therapy as an intervention. We performed a search of electronic databases MEDLINE, Embase, LILACS and SciELO by December 2010. There was no restriction of the period and publication of articles aiming to increase the sensitivity and accuracy; the search strategy used was adapted for each database. We used the score of the PEDro scale for assessing the methodological quality of randomized controlled trials. Results: 26 articles were included in the systematic review, and the evaluation of most of these articles had satisfactory methodological quality (6.4 points). Regarding the aspects measured, all articles evaluated pain, 18 (69\%) examined the grip strength and 11 (42\%) examined the patient's function. The duration of follow-up, only nine (35\%) articles made long-term monitoring. Conclusion: The best option for the treatment of lateral epicondylitis seems to be a combination of therapeutic modalities, which agrees with clinical reality of the physiotherapists. However, more clinical trials with good methodological quality are needed to determine the effectiveness of most therapeutic modalities found.

Keywords: Tennis elbow. Rehabilitation. Physiotherapy.

\section{Introdução}

Ainda hoje existem muitas controvérsias a respeito da epicondilite lateral, desde a sua correta nomenclatura até a verdadeira etiologia, fisiopatologia e seu tratamento ideal (1). Trata-se de uma das patologias de maior facilidade de diagnóstico, porém, de maior dificuldade de tratamento, por mais paradoxal que isso possa parecer (2). A epicondilite lateral geralmente acomete pessoas entre 30 e 60 anos (2-5) e é definida como uma afecção degenerativa que ocorre inicialmente por microlesões na origem da musculatura extensora do antebraço $(3,4,6)$. Sua apresentação clínica caracteriza-se por dor sobre o epicôndilo lateral, com irradiação para a musculatura extensora e diminuição da força de preensão, afetando as atividades cotidianas $(3,4,7)$. Seu mecanismo de lesão se dá por esforços repetitivos, geralmente laborais ou esportivos $(4,5,8)$.

A epicondilite lateral tem um impacto importante na sociedade, desafiando diariamente os clínicos e a indústria terapêutica $(4,9)$. Embora o tratamento conservador ainda seja a melhor conduta inicial para a epicondilite lateral do cotovelo (10), já que resulta em cura em $80 \%$ dos casos, ele é bastante contraditório e discutido (2). Boyer e Hastings (7), em 1999, afirmaram haver pouca evidência científica de que qualquer forma de tratamento não cirúrgico altere o curso natural da doença, que geralmente tem resolução espontânea em um ano. Mais de 40 modalidades terapêuticas conservadoras têm sido relatadas na literatura $(3,4,9,11)$, porém, nenhuma têm se mostrado muito efetiva no tratamento da epicondilite lateral.

Algumas revisões sistemáticas $(8,12,13)$ foram realizadas avaliando os efeitos dessas intervenções fisioterapêuticas, e todas concluíram que apesar de existir um grande número de estudos, não há evidência suficiente que justifique o uso dessas intervenções no tratamento da epicondilite lateral. No entanto, a revisão mais atual (8) reviu os artigos publicados até 2003, e desde então o número de trabalhos realizados sobre esse tema aumentou muito, o que justifica a realização desta revisão sistemática atualizada. Sendo assim, o objetivo desta pesquisa foi avaliar a efetividade e a segurança das 
diversas intervenções fisioterapêuticas utilizadas no tratamento conservador da epicondilite lateral, verificando se há na literatura evidência suficiente para determinar quais intervenções são eficazes no manejo dessa patologia para que se possa criar diretrizes de tratamento.

\section{Materiais e métodos}

\section{Critérios de inclusão}

Foram incluídos apenas ensaios clínicos aleatorizados e quase aleatorizados, que avaliaram adultos com diagnóstico de epicondilite lateral estabelecido por aumento da dor à palpação do epicôndilo lateral e dorsiflexão resistida do punho. Os estudos incluídos precisavam ter pelo menos uma modalidade fisioterapêutica como uma das intervenções no tratamento da epicondilite lateral. Estudos que compararam o uso de uma intervenção fisioterapêutica com injeções de corticoide e medicamentos também serão incluídos no estudo, porém, aqueles que compararam com intervenções cirúrgicas não serão incluídos.

\section{Estratégia de busca (Anexo I)}

Foi realizada uma busca nas bases de dados eletrônicas MEDLINE via PubMed; Embase; LILACS e SciELO até dezembro de 2010. Não houve restrição ao período de publicação e os estudos foram restritos aos idiomas inglês, espanhol e português. Com o objetivo de aumentar a sensibilidade e a precisão da busca, a estratégia utilizada foi adaptada para cada base de dados, sendo explorada de acordo com as características das bases. Também foi realizada uma busca nas referências dos artigos encontrados e anais de congressos.

\section{Seleção dos estudos}

Potenciais estudos identificados pelas estratégias de buscas foram avaliados e selecionados para inclusão na revisão, inicialmente, por meio da leitura dos títulos e resumos. Os textos completos dos potenciais artigos que foram escolhidos pelos títulos e resumos foram acessados e avaliados, aqueles que preencheram os critérios foram selecionados para inclusão na revisão.
Avaliação de qualidade

Foi utilizada a pontuação da escala PEDro para avaliação da qualidade metodológica dos ensaios clínicos aleatorizados já realizada pelos avaliadores e disponível na base de dados PEDro (Physiotherapy Evidence Database). Essa escala consiste em 11 itens, sendo que cada item é pontuado em sim (1 ponto) ou não (0 pontos), e a pontuação final é dada pela soma dos itens definidos como "sim". 0 item 1, critério de elegibilidade, não entra na pontuação final, portanto, a pontuação máxima para cada artigo é dez. Os outros itens são: 2) alocação randômica; 3) ocultação de alocação; 4) comparação no baseline; 5) cegamento dos participantes; 6) cegamento dos terapeutas; 7) cegamento dos avaliadores; 8) seguimento adequado; 9) análise por intenção de tratar; 10) comparação entre os grupos; 11) estimativa de efeito e variabilidade.

\section{Resultados}

Após a coleta dos artigos científicos referentes ao tratamento conservador da epicondilite lateral, foi encontrado um total de 162 artigos. Após a leitura dos títulos e resumos foram excluídos 120 estudos. Com a leitura dos textos completos, 16 foram excluídos por não preencherem os critérios de inclusão do estudo, restando um total de 26 artigos que foram incluídos nesta revisão sistemática (Figura 1). Os 26 artigos selecionados encontram-se resumidos no Quadro 1.

Em relação aos aspectos mensurados, todos os artigos avaliaram a dor com base na escala visual análoga ou em pontos ordinais, 18 (69\%) examinaram a força de preensão, 11 (42\%) a função dos pacientes e dez (38\%) avaliaram a taxa de sucesso através da escala de satisfação do paciente ou melhora global. Quanto à duração do acompanhamento dos pacientes, 17 (65\%) artigos selecionados avaliaram a eficácia dos tratamentos a curto e médio prazo, até seis semanas, e apenas nove (35\%) a longo prazo, ou seja, igual ou maior que seis meses.

No que se refere aos tipos de modalidades terapêuticas utilizadas nos 26 estudos, foi encontrado que 18 (69\%) utilizaram intervenções eletroterapêuticas, sendo que cinco (19\%) avaliaram ondas de choque, cinco (19\%) a terapia por laser, três (11,5\%) o ultrassom, dois (85) a fonoforese e três (11,5\%) a iontoforese. Já para as intervenções não eletroterapêuticas foram encontrados 13 (50\%) artigos dos 26 selecionados, visto que quatro 


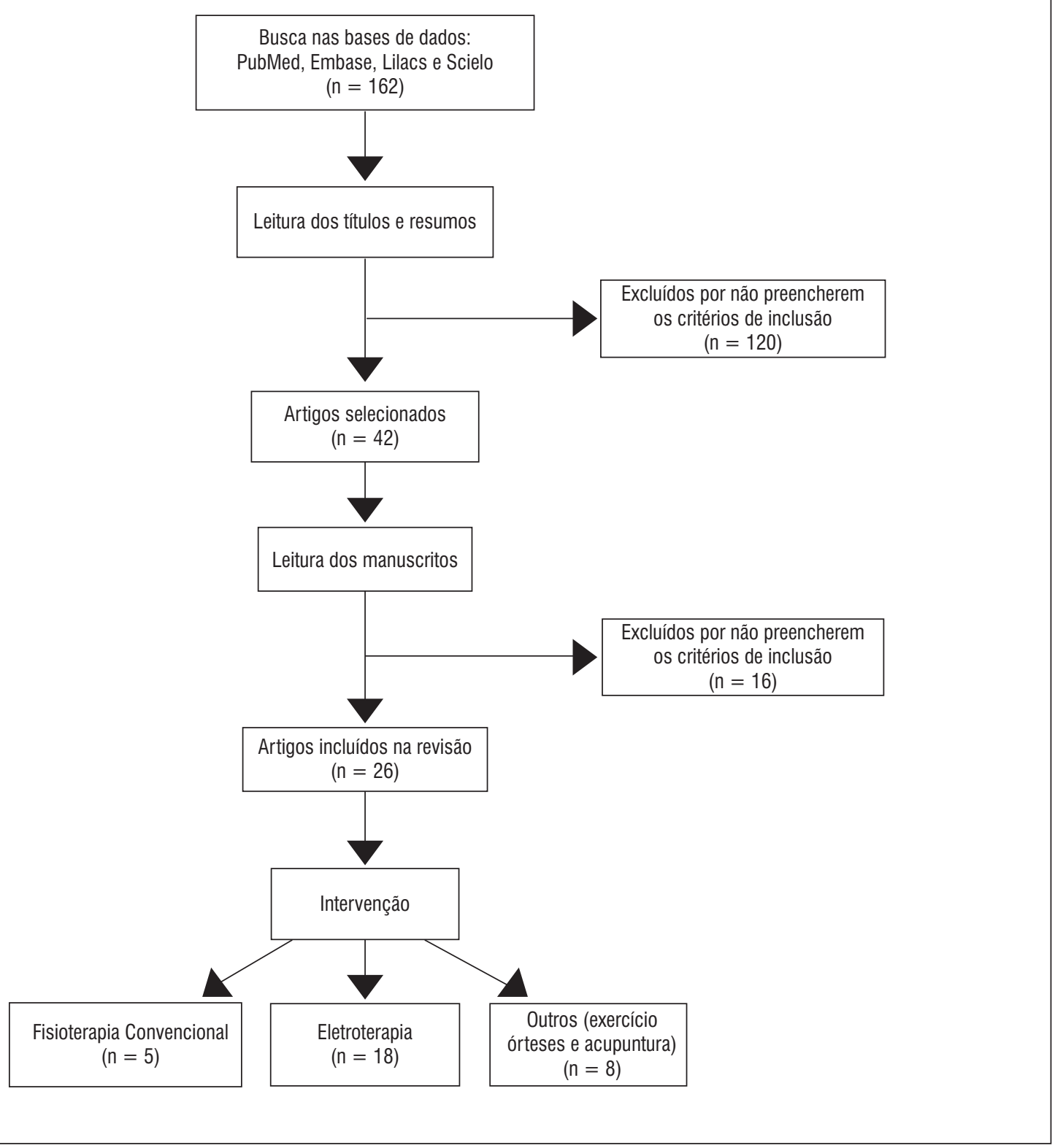

Figura 1 - Fluxograma do processo de inclusão dos artigos na revisão sistemática Fonte: Dados da pesquisa.

(15\%) examinaram os exercícios terapêuticos, um (4\%) a técnica manipulativa, dois (8\%) o uso de órtese, um (4\%) a acupuntura e cinco (19\%) avaliaram a eficácia da fisioterapia convencional.

Os resultados da avaliação da qualidade dos artigos incluídos utilizando a escala PEDro demonstraram uma pontuação média de 6,4 pontos (Anexo II). Houve maior perda de pontuação por parte dos artigos nos itens 9 (análise por intenção de tratar), 6 (cegamento dos terapeutas) e 3 (ocultação de alocação). Nenhum artigo apresentou a pontuação máxima na escala. Os artigos que receberam maior pontuação foram os que tratavam do uso da terapia por ondas de choque extracorpórea e fisioterapia convencional.

\section{Discussão}

Os estudos encontrados sobre o tratamento fisioterapêutico da epicondilite lateral estão listados a seguir, de acordo com o tipo de intervenção abordada. 
Fisioterapia convencional

Newcomer et al. (14) realizaram um estudo comparando um grupo que realizou fisioterapia convencional e o outro que realizou fisioterapia convencional acrescido de injeções de corticoide. Os resultados indicaram que a fisioterapia convencional foi responsável pela melhora da maior parte dos pacientes tanto a curto como a longo prazo. Seu protocolo consistiu de fortalecimento progressivo com exercícios excêntricos e concêntricos e aplicação de gelo no epicôndilo lateral. Apesar de os resultados serem favoráveis à fisioterapia, a amostra utilizada foi pequena ( $n=39)$, mesmo apresentando uma boa qualidade metodológica.

Smidt et al. (15) confirmaram os resultados obtidos por Newcomer et al. (14), afirmando que a fisioterapia é o método mais eficaz no tratamento dessa patologia. Os autores utilizaram um protocolo que consistiu na aplicação de ultrassom pulsátil, massagem profunda transversa e exercícios terapêuticos. Bisset et al. (16) encontraram resultados que também confirmam a fisioterapia convencional como um método eficaz no tratamento da epicondilite lateral, apresentando-a como uma alternativa para substituir as injeções de corticoide a médio e longo prazo. A diferença entre os estudos foi que Bisset et al. (16) utilizaram um protocolo que consistia de oito sessões de manipulação do cotovelo e exercícios terapêuticos. Struijs et al. (17) também relataram a eficácia da fisioterapia, principalmente a curto prazo, comparada com o uso do brace e com a combinação de ambos os tratamentos. 0 protocolo da fisioterapia consistiu de ultrassom pulsátil, massagem profunda transversa e exercícios terapêuticos.

\section{Exercícios terapêuticos}

Quatro estudos (18-21) analisaram a eficácia do exercício terapêutico no tratamento da epicondilite lateral. Pienimaki et al. (18) realizaram um estudo comparando o uso do programa de exercícios realizados em casa com o ultrassom, em pacientes com epicondilite lateral crônica. 0 programa de exercícios desse estudo consistiu de alongamento, fortalecimento progressivo excêntrico e concêntrico do punho e exercícios ocupacionais. Os autores mostraram que o programa de exercícios utilizado foi efetivo no tratamento a curto prazo da epicondilite lateral crônica, já que, além de aumentar a força muscular, houve melhora na função e um efeito analgésico nos pacientes. Manias e Stasinopoulos (19) também encontraram resultados positivos do programa de exercícios no tratamento a curto prazo da epicondilite lateral. Porém, esse estudo foi qualificado com baixa qualidade metodológica, o que torna os resultados não tão confiáveis.

Tonks et al. (20), em 2006, apresentaram resultados que conflitam com os achados em estudos anteriores $(21,22)$, comparando o uso de injeções de corticoide, exercícios terapêuticos e a combinação de ambos no tratamento da epicondilite lateral aguda. Os resultados indicaram que, após sete semanas, não houve efeitos benéficos dos exercícios terapêuticos em relação à dor e à função. Nagrale et al. (21) também não encontraram resultados positivos no estudo quando comparado com a técnica de terapia manual Cyriax.

\section{Ondas de choque}

Rompe et al. (22), em 2004, obtiveram resultados positivos com a terapia por ondas de choque extracorpórea de baixa intensidade em relação ao grupo placebo no tratamento da epicondilite lateral crônica em jogadores de tênis recreacional, tanto a curto como a longo prazo. Os autores utilizaram um protocolo de três sessões, com uma semana de intervalo entre cada sessão, sendo que cada sessão consistia de uma terapia de 2000 pulsos e uma energia de fluxo de $0,09 \mathrm{~mJ} / \mathrm{mm}^{2}$, não sendo utilizada anestesia local. Melegati et al. (23) confirmaram esses achados, confirmando a eficácia da terapia por ondas de choque de baixa intensidade no tratamento da epicondilite lateral a curto e longo prazo.

Haake et al. (24) falharam ao tentar mostrar alguma eficácia da terapia por ondas de choque de baixa intensidade em pacientes com epicondilite lateral crônica, já que não houve diferenças significativas tanto a curto como a longo prazo. 0 resultado pode ser explicado por alguns fatores que diferem do estudo realizado por Rompe et al. (22), como o uso da anestesia local, a qual parece ser inadequada, o uso de AINH imediatamente durante e após o tratamento e o uso de diferentes doses para cada paciente. Speed et al. (25) apresentaram resultados que não comprovam a eficácia da terapia por ondas de choque de moderada intensidade a curto prazo no tratamento 
da epicondilite lateral crônica. 0 protocolo utilizado foi de três sessões, sendo que cada sessão consistia de 1500 pulsos, um fluxo de energia de $0,18 \mathrm{~mJ} / \mathrm{mm}^{2}$, não sendo utilizada anestesia local.

Chung e Wiley (26), em 2004, também não encontraram diferenças significativas entre os grupos tratados com terapia de ondas de choque extracorpórea (três sessões com 2000 pulsos com energia de fluxo de 0,03 a $0,17 \mathrm{~mJ} / \mathrm{mm}^{2}$ ) e o grupo placebo. Apesar de os resultados estarem de acordo com os estudos anteriores $(22,24,25)$, é difícil realizar comparações, pois esse foi o único estudo em que foram recrutados pacientes que não tinham sido previamente tratados.

Os resultados encontrados a respeito da terapia por ondas de choque extracorpórea foram conflitantes, sendo difícil obter uma conclusão, já que os estudos apresentavam diferentes tipos de protocolo de tratamento, diferentes grupos para comparação e diferentes características clínicas dos pacientes.

\section{Ultrassom}

D’Vaz et al. (27) avaliaram a eficácia da terapia do ultrassom em 48 pacientes com epicondilite lateral crônica, utilizando o modo pulsátil, de baixa intensidade $\left(30 \mathrm{~mW} / \mathrm{cm}^{2}\right)$ e frequência de $1.5 \mathrm{MHz}$. Após o fim do tratamento, não houve diferença significativa entre os grupos. Pienimaki et al. (18) também encontraram resultados desfavoráveis ao uso do ultrassom pulsátil no tratamento a curto prazo em pacientes com epicondilite lateral crônica quando comparados com exercícios. 0 protocolo utilizado foi de $1 \mathrm{MHz}$, modo pulsátil, e intensidade de $0,3 \mathrm{a} 0,7 \mathrm{~W} / \mathrm{cm}^{2}$. Esses achados foram corroborados por Oken et al. (28), que encontraram resultados negativos do ultrassom em relação ao laser e ao brace.

\section{Iontoforese}

Runeson et al. (29), em 2002, demonstraram que a administração de corticoide via iontoforese não foi eficaz no tratamento da epicondilite lateral tanto a curto como a longo prazo. Após três e seis meses, ambos os grupos tratados e placebo apresentaram melhora significativa em relação à dor e à força de preensão. Resultados diferentes foram encontrados por Nirschl et al. (30), em 2003, que obtiveram resultados favoráveis a curto prazo no tratamento da epicondilite lateral: $52 \%$ dos pacientes do grupo experimental obtiveram uma melhora moderada ou maior após o fim do tratamento, comparada com 33\% do grupo placebo. A melhora observada nesse estudo pode ser explicada pelas características clínicas dos pacientes, que tinham sintomas com intensidade moderada a grave.

\section{Laser}

Os cinco estudos selecionados apresentaram resultados conflitantes. Basford et al. (31) obtiveram resultados que não justificaram o uso do laser de baixa intensidade no tratamento da epicondilite lateral, já que não houve diferenças significativas em relação ao grupo placebo. Isso pode ser explicado, talvez, porque os parâmetros utilizados não sejam os mais adequados, já que se trata de uma disfunção superficial e os autores utilizaram um comprimento de onda de 1.06 micromilímetros, dosagem de $12.34 \mathrm{~J} / \mathrm{cm}^{2}$. Oken et al. (28) encontraram resultados favoráveis para o uso do laser na força de preensão comparado ao uso do brace, porém, para a variável dor, o laser não foi eficaz.

Ogueta (32), em um estudo realizado em 2000, obteve resultados favoráveis com o uso da terapia por laser de baixa intensidade no tratamento da epicondilite lateral a curto prazo. Após o fim do tratamento, o grupo experimental apresentou diminuição de dor em $45 \%$ dos pacientes, comparado com $17 \%$ do placebo. Os autores utilizaram no protocolo um comprimento de onda de 830 micromilímetros, dosagem de $2 \mathrm{~J} / \mathrm{cm}^{2}$ e uma aplicação pontual de quatro a seis pontos. 0 protocolo utilizado foi mais adequado que o estudo realizado por Basford et al. (31), que utilizaram um comprimento de onda grande e uma dosagem alta. Resultados favoráveis também foram encontrados nos estudos de Lam et al. (33) e Stergioulas (34), com um protocolo parecido com o estudo de Gueta, que demonstraram mais eficácia do laser em comparação com o placebo para as variáveis dor, força de preensão e função. Esses estudos demonstraram que a terapia por laser pode ser utilizada no tratamento da epicondilite lateral.

Este estudo apresenta como limitação o uso de apenas três idiomas para o recrutamento dos artigos, inglês, espanhol e português, além da utilização de quatro bases de dados. Talvez nem todos os ensaios clínicos publicados sobre o tema estejam inclusos. 
Quadro 1 - Características dos artigos incluídos

Continua

\begin{tabular}{|c|c|c|c|c|}
\hline Estudo & Delineamento & População & Variáveis & Resultados \\
\hline Smidt et al., (15) & $\begin{array}{l}\text { Ensaio clínico } \\
\text { randomizado e } \\
\text { controlado }\end{array}$ & $\begin{array}{l}185 \text { pacientes } \\
\text { com epicondilite } \\
\text { lateral (duração } \\
\text { dos sintomas de no } \\
\text { mínimo seis semanas) } \\
\text { foram recrutados e } \\
\text { randomizados em três } \\
\text { grupos. }\end{array}$ & $\begin{array}{l}\text { Injeções de } \\
\text { corticoide x } \\
\text { fisioterapia } \\
\text { convencional x } \\
\text { controle }\end{array}$ & $\begin{array}{l}\text { Após seis semanas, as injeções de } \\
\text { corticoide apresentaram } 92 \% \text { de sucesso } \\
\text { comparadas com } 47 \% \text { da fisioterapia } \\
\text { e } 32 \% \text { do grupo controle. Já após } 52 \\
\text { semanas, o sucesso das injeções de } \\
\text { corticoide caiu para } 69 \% \text {, enquanto na } \\
\text { fisioterapia aumentou para } 91 \% \text { e no } \\
\text { controle para } 83 \% \text {. }\end{array}$ \\
\hline Bisset et al., (16) & $\begin{array}{l}\text { Ensaio clínico } \\
\text { randomizado, } \\
\text { controlado e } \\
\text { duplo-cego }\end{array}$ & $\begin{array}{l}198 \text { pacientes com } \\
\text { epicondilite lateral } \\
\text { (duração dos sintomas } \\
\text { de no mínimo seis } \\
\text { semanas) foram } \\
\text { selecionados e divididos } \\
\text { em três grupos. }\end{array}$ & $\begin{array}{l}\text { Injeções de } \\
\text { corticoide x } \\
\text { fisioterapia } \\
\text { convencional } x \\
\text { controle }\end{array}$ & $\begin{array}{l}\text { Após seis semanas, o grupo que usou } \\
\text { corticoide obteve mais sucesso }(78 \%) \text { que } \\
\text { a fisioterapia (65\%) e o placebo ( } 27 \%) \text {. } \\
\text { Ao final de } 52 \text { semanas, as injeções de } \\
\text { corticoide apresentaram } 68 \% \text { de sucesso, } \\
\text { enquanto que a fisioterapia obteve } 94 \% \text { e } 0 \\
\text { placebo } 90 \% \text {. }\end{array}$ \\
\hline $\begin{array}{l}\text { Newcomer } \\
\text { et al., (14) }\end{array}$ & $\begin{array}{l}\text { Ensaio clínico } \\
\text { randomizado, } \\
\text { controlado e } \\
\text { duplo-cego }\end{array}$ & $\begin{array}{l}39 \text { pacientes com } \\
\text { epicondilite lateral } \\
\text { (sintomas com } \\
\text { duração menor que } \\
\text { quatro semanas) } \\
\text { foram selecionados e } \\
\text { randomizados em dois } \\
\text { grupos. }\end{array}$ & $\begin{array}{l}\text { Injeção de } \\
\text { corticoide + } \\
\text { fisioterapia } \\
\text { convencional } \\
\text { x fisioterapia } \\
\text { convencional }\end{array}$ & $\begin{array}{l}\text { Após seis meses do início do tratamento, } \\
\text { ambos os grupos apresentaram melhora } \\
\text { significativa em mais de } 80 \% \text { em relação à } \\
\text { dor, força e função. Porém, sem diferenças } \\
\text { significativas entre os grupos até o } 6^{\circ} \\
\text { mês, em que houve melhora significativa } \\
\text { no grupo experimental. }\end{array}$ \\
\hline Strujis et al., (17) & $\begin{array}{l}\text { Ensaio clínico } \\
\text { randomizado e } \\
\text { piloto }\end{array}$ & $\begin{array}{l}28 \text { pacientes com } \\
\text { epicondilite lateral } \\
\text { (duração dos sintomas } \\
\text { de no mínimo seis } \\
\text { semanas) foram } \\
\text { escolhidos para } \\
\text { participar do estudo. }\end{array}$ & $\begin{array}{l}\text { Manipulação } \\
\text { de punhox } \\
\text { fisioterapia } \\
\text { convencional }\end{array}$ & $\begin{array}{l}\text { Após três semanas, a manipulação do } \\
\text { punho obteve sucesso em } 62 \% \text { dos } \\
\text { pacientes, enquanto que a fisioterapia } \\
\text { convencional apresentou sucesso em } \\
20 \% \text { dos pacientes. Com seis semanas, } \\
\text { a manipulação do punho obteve sucesso } \\
\text { em } 85 \% \text { dos pacientes contra } 67 \% \text { da } \\
\text { fisioterapia convencional. }\end{array}$ \\
\hline Tonks et al., (20) & $\begin{array}{l}\text { Ensaio clínico } \\
\text { randomizado, } \\
\text { controlado e } \\
\text { prospectivo }\end{array}$ & $\begin{array}{l}36 \text { pacientes com } \\
\text { epicondilite lateral (não } \\
\text { tinham sido tratados } \\
\text { nos últimos seis meses) } \\
\text { foram randomizados em } \\
\text { três grupos. }\end{array}$ & $\begin{array}{l}\text { Injeções de } \\
\text { corticoide } x \\
\text { exercícios x } \\
\text { ambos }\end{array}$ & $\begin{array}{l}\text { Após sete semanas, as injeções } \\
\text { de corticoide obtiveram resultado } \\
\text { significativamente positivo em relação à } \\
\text { força de preensão, dor e função. }\end{array}$ \\
\hline Garg et al., (35) & $\begin{array}{l}\text { Ensaio clínico } \\
\text { randomizado, } \\
\text { controlado, } \\
\text { prospectivo }\end{array}$ & $\begin{array}{l}42 \text { pacientes com } \\
\text { epicondilite lateral } \\
\text { aguda. }\end{array}$ & $\begin{array}{l}\text { Splint (punho) } x \\
\text { brace (antebraço) }\end{array}$ & $\begin{array}{l}\text { Não houve diferença significativa entre } \\
\text { os grupos em relação à função. Já para } \\
\text { a dor o grupo que usou splint apresentou } \\
\text { melhora da dor em relação ao grupo que } \\
\text { usou o brace. }\end{array}$ \\
\hline Struijs et al., (17) & $\begin{array}{l}\text { Ensaio clínico } \\
\text { randomizado } \\
\text { controlado }\end{array}$ & $\begin{array}{l}180 \text { pacientes com } \\
\text { epicondilite lateral (com } \\
\text { duração dos sintomas de } \\
\text { no mínimo seis semanas) } \\
\text { foram selecionados e } \\
\text { randomizados em três } \\
\text { grupos. }\end{array}$ & $\begin{array}{l}\text { Brace } x \text { fisioterapia } \\
\text { convencional x } \\
\text { ambos }\end{array}$ & $\begin{array}{l}\text { A curto prazo (seis semanas), a fisioterapia } \\
\text { apresentou } 50 \% \text { de sucesso, comparada com } \\
45 \% \text { do protocolo combinado e } 40 \% \text { do brace. } \\
\text { A médio ( } 26 \text { semanas) e longo prazo ( } 52 \text { sema- } \\
\text { nas) não houve diferenças entre os grupos. }\end{array}$ \\
\hline Manias et al., (19) & $\begin{array}{l}\text { Ensaio clínico } \\
\text { monocêntrico }\end{array}$ & $\begin{array}{l}40 \text { pacientes com } \\
\text { epicondilite lateral (com } \\
\text { duração dos sintomas de } \\
\text { no mínimo quatro semanas) } \\
\text { foram selecionados e } \\
\text { divididos em dois grupos. }\end{array}$ & $\begin{array}{l}\text { Exercícios } x \\
\text { exercícios }+ \text { gelo } \\
\text { ) }\end{array}$ & $\begin{array}{l}\text { Após o fim do tratamento (quatro semanas) } \\
\text { houve um declínio significativo (sete unidades } \\
\text { na EVA) em ambos os grupos. Após } 16 \\
\text { semanas esses resultados se mantiveram. }\end{array}$ \\
\hline
\end{tabular}


Quadro 1 - Características dos artigos incluídos

Continuação

\begin{tabular}{|c|c|c|c|c|}
\hline Estudo & Delineamento & População & Variáveis & Resultados \\
\hline $\begin{array}{l}\text { Park et } \\
\text { al., (36) }\end{array}$ & $\begin{array}{l}\text { Ensaio clínico } \\
\text { randomizado, } \\
\text { controlado, } \\
\text { prospectivo }\end{array}$ & $\begin{array}{l}31 \text { pacientes com } \\
\text { epicondilite lateral aguda. }\end{array}$ & $\begin{array}{l}\text { Exercícios (imediato) x exercícios } \\
\text { (após quatro semanas) }\end{array}$ & $\begin{array}{l}0 \text { grupo que realizou exercícios } \\
\text { imediatamente apresentou } \\
\text { redução significativa da dor } \\
\text { após um mês de seguimento } \\
\text { em relação ao grupo controle. } \\
\text { Essa diferença não se manteve } \\
\text { após } 3,6 \text { e } 12 \text { meses de } \\
\text { seguimento. }\end{array}$ \\
\hline $\begin{array}{l}\text { Pienimaki } \\
\text { et al., (18) }\end{array}$ & $\begin{array}{l}\text { Ensaio clínico } \\
\text { randomizado }\end{array}$ & $\begin{array}{l}39 \text { pacientes com } \\
\text { epicondilite lateral crônica } \\
\text { (duração dos sintomas de } \\
\text { no mínimo três meses) } \\
\text { foram randomizados em } \\
\text { dois grupos de tratamento. }\end{array}$ & Ultrassom pulsado $\mathrm{x}$ exercícios & $\begin{array}{l}\text { Em oito semanas de tratamento, } \\
\text { diminuição significativa na dor } \\
\text { no grupo de exercício. Torque de } \\
\text { flexão de punho aumentou } 45 \% \\
\text { no grupo do exercício e a força } \\
\text { de preensão aumentou } 12 \% \text { no } \\
\text { grupo exercício. }\end{array}$ \\
\hline $\begin{array}{l}\text { D'Vaz et } \\
\text { al., (27) }\end{array}$ & $\begin{array}{l}\text { Ensaio clínico } \\
\text { randomizado, e } \\
\text { duplo-cego }\end{array}$ & $\begin{array}{l}48 \text { pacientes com } \\
\text { epicondilite lateral crônica } \\
\text { (duração dos sintomas de } \\
\text { no mínimo seis semanas) } \\
\text { foram randomizados em } \\
\text { dois grupos. }\end{array}$ & Ultrassom pulsado $\mathrm{x}$ exercícios & $\begin{array}{l}\text { Não houve diferença significante } \\
\text { entre os grupos para a dor e } \\
\text { função após } 12 \text { semanas. }\end{array}$ \\
\hline $\begin{array}{l}\text { Nagrale et } \\
\text { al., (21) }\end{array}$ & $\begin{array}{l}\text { Ensaio clínico } \\
\text { randomizado, } \\
\text { controlado }\end{array}$ & $\begin{array}{l}60 \text { pacientes com } \\
\text { epicondilite lateral, com } \\
\text { duração dos sintomas de } \\
\text { pelo menos um mês. }\end{array}$ & Cyriax x fonoforese + exercícios & $\begin{array}{l}\text { Ambos os grupos melhorarem } \\
\text { após o tratamento. } 0 \text { grupo } \\
\text { que realizou Cyriax apresentou } \\
\text { melhores resultados em relação } \\
\text { à dor, força de preensão e } \\
\text { função. }\end{array}$ \\
\hline $\begin{array}{l}\text { Baskurt et } \\
\text { al., (37) }\end{array}$ & $\begin{array}{l}\text { Ensaio clínico } \\
\text { randomizado }\end{array}$ & $\begin{array}{l}61 \text { pacientes com } \\
\text { epicondilite lateral } \\
\text { foram selecionados e } \\
\text { randomizados em dois } \\
\text { grupos. }\end{array}$ & $\begin{array}{l}\text { Iontoforese (naproxeno) x fonoforese } \\
\text { (naproxeno) }\end{array}$ & $\begin{array}{l}\text { A dor diminuiu em ambos os } \\
\text { grupos. A força de preensão e } \\
\text { o nível de função melhoraram } \\
\text { nos dois grupos, sem diferenças } \\
\text { significativas entre os grupos. }\end{array}$ \\
\hline $\begin{array}{l}\text { Nirschl et } \\
\text { al., (30) }\end{array}$ & $\begin{array}{l}\text { Ensaio clínico } \\
\text { randomizado e } \\
\text { duplo-cego }\end{array}$ & $\begin{array}{l}199 \text { pacientes com } \\
\text { epicondilite lateral } \\
\text { randomizados em dois } \\
\text { grupos. }\end{array}$ & Iontoforese (corticoide) x placebo & $\begin{array}{l}0 \text { grupo experimental apresentou } \\
\text { melhora significativa na dor } \\
\text { em comparação com o grupo } \\
\text { controle. }\end{array}$ \\
\hline $\begin{array}{l}\text { Runeson } \\
\text { e Haker, } \\
\text { (29) }\end{array}$ & $\begin{array}{l}\text { Ensaio clínico } \\
\text { randomizado, } \\
\text { controlado, } \\
\text { duplo-cego e } \\
\text { prospectivo }\end{array}$ & $\begin{array}{l}64 \text { pacientes com } \\
\text { epicondilite lateral } \\
\text { (sintomas com duração } \\
\text { de no mínimo um mês) } \\
\text { randomizados em dois } \\
\text { grupos. }\end{array}$ & Iontoforese (corticoide) x placebo & $\begin{array}{l}\text { Não se observou melhora } \\
\text { entre os grupos em relação à } \\
\text { dor a curto prazo. Após três e } \\
\text { seis meses ambos os grupos } \\
\text { tiveram melhora significativa } \\
\text { em relação à dor e força de } \\
\text { preensão com uma taxa de } \\
\text { sucesso de } 80 \% \text {. }\end{array}$ \\
\hline $\begin{array}{l}\text { Fink et al., } \\
\text { (38) }\end{array}$ & $\begin{array}{l}\text { Ensaio clínico } \\
\text { controlado, } \\
\text { randomizado e } \\
\text { simples-cego }\end{array}$ & $\begin{array}{l}45 \text { pacientes com } \\
\text { epicondilite lateral crônica } \\
\text { foram selecionados e } \\
\text { randomizados em dois } \\
\text { grupos. }\end{array}$ & Acupuntura $x$ placebo & $\begin{array}{l}\text { Após duas semanas do } \\
\text { fim do tratamento, o grupo } \\
\text { tratado apresentou resultados } \\
\text { significantemente melhores que } \\
\text { o placebo em relação à dor, } \\
\text { força e incapacidade funcional. }\end{array}$ \\
\hline $\begin{array}{l}\text { Ogueta, } \\
(32)\end{array}$ & $\begin{array}{l}\text { Ensaio clínico } \\
\text { prospectivo, } \\
\text { simples-cego }\end{array}$ & $\begin{array}{l}86 \text { pacientes com } \\
\text { epicondilite lateral aguda } \\
\text { e subaguda foram } \\
\text { selecionados e divididos } \\
\text { em dois grupos. }\end{array}$ & Laser x placebo & $\begin{array}{l}0 \text { grupo experimental apresentou } \\
\text { diminuição da dor em } 45 \% \text { dos } \\
\text { pacientes, comparado com } 17 \% \\
\text { do placebo. }\end{array}$ \\
\hline
\end{tabular}


Quadro 1 - Características dos artigos incluídos

\begin{tabular}{|c|c|c|c|c|}
\hline Estudo & Delineamento & População & Variáveis & Resultados \\
\hline $\begin{array}{l}\text { Basford et } \\
\text { al., (31) }\end{array}$ & $\begin{array}{l}\text { Ensaio clínico } \\
\text { randomizado, } \\
\text { controlado e } \\
\text { duplo-cego }\end{array}$ & $\begin{array}{l}52 \text { pacientes com } \\
\text { epicondilite lateral (com } \\
\text { duração dos sintomas } \\
\text { maior que } 30 \text { dias) foram } \\
\text { selecionados e divididos } \\
\text { em dois grupos. }\end{array}$ & Laser $\mathrm{x}$ placebo & $\begin{array}{l}\text { Não houve diferenças entre } \\
\text { os grupos nas variáveis: dor, } \\
\text { função, sensibilidade, força de } \\
\text { preensão, função do braço, uso de } \\
\text { medicamentos e uso de órtese. }\end{array}$ \\
\hline $\begin{array}{l}\text { Lam et al., } \\
\text { (33) }\end{array}$ & $\begin{array}{l}\text { Ensaio clínico } \\
\text { randomizado, } \\
\text { prospectivo }\end{array}$ & $\begin{array}{l}39 \text { pacientes com } \\
\text { epicondilite lateral } \\
\text { crônica, com duração dos } \\
\text { sintomas de no mínimo } \\
\text { três meses. }\end{array}$ & Laser $\mathrm{x}$ placebo & $\begin{array}{l}\text { Melhora significativa do grupo } \\
\text { experimental comparado com o } \\
\text { placebo em relação à dor, limiar de } \\
\text { dor a palpação, força de preensão } \\
\text { e função após três semanas de } \\
\text { tratamento e após três semanas do } \\
\text { término do tratamento. }\end{array}$ \\
\hline $\begin{array}{l}\text { Stergioulas, } \\
\text { (34) }\end{array}$ & $\begin{array}{l}\text { Ensaio clínico } \\
\text { randomizado e } \\
\text { simples-cego }\end{array}$ & $\begin{array}{l}50 \text { pacientes com } \\
\text { epicondilite lateral } \\
\text { aguda, com duração dos } \\
\text { sintomas maior que cinco } \\
\text { semanas. }\end{array}$ & $\begin{array}{l}\text { Laser + pliometria } x \text { laser } \\
\text { (placebo) + pliometria }\end{array}$ & $\begin{array}{l}\text { Melhora significativa do grupo experi- } \\
\text { mental comparado com o controle em } \\
\text { relação à dor, força de preensão e ampli- } \\
\text { tude de movimento após oito semanas } \\
\text { de tratamento. }\end{array}$ \\
\hline $\begin{array}{l}\text { Oken et al., } \\
(28)\end{array}$ & $\begin{array}{l}\text { Ensaio clínico } \\
\text { randomizado, } \\
\text { prospectivo }\end{array}$ & $\begin{array}{l}52 \text { pacientes com } \\
\text { epicondilite lateral com } \\
\text { duração dos sintomas de, } \\
\text { no mínimo, sete dias. }\end{array}$ & Laser $\mathrm{x}$ brace $\mathrm{x}$ ultrassom & $\begin{array}{l}0 \text { brace apresentou resultados um } \\
\text { pouco melhores do que o laser e } \\
0 \text { ultrassom em relação à dor. Em } \\
\text { relação à força de preensão, o laser foi } \\
\text { mais eficaz que o brace e } 0 \text { ultrassom. }\end{array}$ \\
\hline $\begin{array}{l}\text { Rompe et } \\
\text { al., (22) }\end{array}$ & $\begin{array}{l}\text { Ensaio clínico } \\
\text { randomizado, } \\
\text { controlado e } \\
\text { duplo-cego }\end{array}$ & $\begin{array}{l}78 \text { pacientes com } \\
\text { epicondilite lateral crônica } \\
\text { (duração dos sintomas de } \\
\text { pelo menos um ano) foram } \\
\text { divididos em dois grupos. }\end{array}$ & Ondas de choque $\mathrm{x}$ placebo & $\begin{array}{l}\text { Após três meses do término do } \\
\text { tratamento, } 58 \% \text { dos pacientes do grupo } \\
\text { tratado atingiram resultados excelentes } \\
\text { ou bons comparados com } 33 \% \text { do } \\
\text { grupo controle. Após um ano, foram } \\
63 \% \text { do grupo tratado e } 43 \% \text { do grupo } \\
\text { controle. }\end{array}$ \\
\hline $\begin{array}{l}\text { Haake et al., } \\
\text { (24) }\end{array}$ & $\begin{array}{l}\text { Ensaio clínico } \\
\text { randomizado, } \\
\text { controlado, } \\
\text { prospectivo e } \\
\text { simples-cego }\end{array}$ & $\begin{array}{l}272 \text { pacientes com } \\
\text { epicondilite lateral crônica } \\
\text { (duração dos sintomas } \\
\text { de aproximadamente dois } \\
\text { anos) foram divididos em } \\
\text { dois grupos. }\end{array}$ & Ondas de choque $\mathrm{x}$ placebo & $\begin{array}{l}\text { Após seis semanas, } 27,2 \% \\
\text { dos pacientes do grupo tratado } \\
\text { apresentaram sucesso no tratamento, } \\
\text { comparado com } 23,2 \% \text { do grupo } \\
\text { placebo. } \\
\text { Com } 12 \text { meses, o grupo tratado } \\
\text { obteve } 65,7 \% \text { e o placebo } 65,3 \% \text {. }\end{array}$ \\
\hline $\begin{array}{l}\text { Speed et al., } \\
\text { (25) }\end{array}$ & $\begin{array}{l}\text { Ensaio clínico } \\
\text { randomizado, } \\
\text { controlado e } \\
\text { duplo-cego }\end{array}$ & $\begin{array}{l}75 \text { pacientes com } \\
\text { epicondilite lateral crônica } \\
\text { foram randomizados em } \\
\text { grupos: grupo experimental } \\
\text { e grupo controle. }\end{array}$ & Ondas de choque $\mathrm{x}$ placebo & $\begin{array}{l}\text { Ambos os grupos apresentaram melhora } \\
\text { significativa em relação à dor. Em } \\
\text { relação à dor noturna, } 30 \% \text { do grupo } \\
\text { tratado e } 43 \% \text { do placebo obtiveram } \\
\text { melhora significativa. }\end{array}$ \\
\hline $\begin{array}{l}\text { Chung e } \\
\text { Wiley, (26) }\end{array}$ & $\begin{array}{l}\text { Ensaio clínico } \\
\text { randomizado, } \\
\text { controlado e } \\
\text { duplo-cego }\end{array}$ & $\begin{array}{l}60 \text { pacientes com } \\
\text { epicondilite lateral } \\
\text { previamente não tratada } \\
\text { foram randomizados em } \\
\text { dois grupos. }\end{array}$ & Ondas de choque $\mathrm{x}$ placebo & $\begin{array}{l}\text { Houve melhora significativa em ambos } \\
\text { os grupos em relação à dor e função. } \\
\text { Não houve diferença significativa entre } \\
\text { os grupos. }\end{array}$ \\
\hline $\begin{array}{l}\text { Melegati et } \\
\text { al.,(23) }\end{array}$ & $\begin{array}{l}\text { Ensaio clínico } \\
\text { randomizado }\end{array}$ & $\begin{array}{l}41 \text { pacientes com epicon- } \\
\text { dilite lateral (sintomas com } \\
\text { duração de no mínimo três } \\
\text { meses) foram seleciona- } \\
\text { dos e randomizados em } \\
\text { dois grupos. }\end{array}$ & $\begin{array}{l}\text { Ondas de choque (lateral) } x \\
\text { ondas de choque (posterior) }\end{array}$ & $\begin{array}{l}\text { Não houve diferenças significativas } \\
\text { entre os grupos. Ambos os grupos } \\
\text { apresentaram melhora em relação à dor } \\
\text { e à função. }\end{array}$ \\
\hline
\end{tabular}

Fonte: Dados da pesquisa. 


\section{Conclusão}

A análise dos artigos selecionados neste trabalho de revisão de literatura demonstrou divergências na literatura científica a respeito do tratamento fisioterapêutico da epicondilite lateral. A maioria dos artigos relacionados possuía qualidade metodológica satisfatória, porém, apresentou algumas falhas, como o não acompanhamento a longo prazo, tamanho pequeno da amostra, ausência de blindagem e falta de descrição de como os pacientes foram selecionados.

Apesar das limitações dos artigos, é possível afirmar que a melhor opção para o tratamento da epicondilite lateral é a combinação de modalidades terapêuticas, o que condiz com a realidade clínica do fisioterapeuta, que geralmente não realiza somente um tipo de tratamento. A combinação de exercícios terapêuticos, ultrassom pulsátil e massagem profunda transversa parece ser uma boa opção para o tratamento da epicondilite lateral. 0 uso de modalidades terapêuticas como uma única opção de tratamento, como o ultrassom, o laser, a iontoforese e ondas de choque, não mostraram evidências de benefícios tanto a curto como a longo prazo.

\section{Referências}

1. Cohen M, Romeo A. Lateral Epicondilitis: open and arthroscopic treatment. J Am Soc Surg Hand. 2001;1(3):172-76.

2. Lech O, Piluski P, Severo A. Epicondilite lateral do cotovelo. Rev Bras Ortop. 2003;38(8):421-36.

3. Hong QN, Durand MJ, Loisel P. Treatment of lateral epicondylitis: where is the evidence? Joint Bone Spine. 2004;71(5):369-73.

4. Stasinopoulos D, Johnson MI. Cyriax physiotherapy for tennis elbow/lateral epicondylitis. Br J Sports Med. 2004;38(6):675-7.

5. Stasinopoulos D, Johnson MI. Effectiveness of extracorporeal shock wave therapy for tennis elbow (lateral epicondylitis). Br J Sports Med. 2005;39(3):132-6.

6. Cohen M. Epicondilite lateral do cotovelo. R Into. 2004;2(3):5-12.

7. Boyer MI, Hastings H. Lateral tennis elbow: "Is there any science out there?". J Shoulder Elbow Surg. 1999;8(5):481-91.
8. Bisset L, Paungmali A, Vicenzino B, Beller E. A systematic review and meta-analysis of clinical trials on physical interventions for lateral epicondylalgia. Br J Sports Med. 2005;39(7):411-22; discussion -22.

9. Sevier TL, Wilson JK. Treating lateral epicondylitis. Sports Med. 1999;28(5):375-80.

10. Coelho SP, Franco N Filho, Bastos LC, Oliveira SB. Epicondilite lateral do úmero: tratamento conservador. Um estudo de 28 casos. Acta Ortop Bras. 1996;4(4):1-3.

11. Struijs PA, Smidt N, Arola H, van Dijk CN, Buchbinder R, Assendelft WJ. Orthotic devices for tennis elbow: a systematic review. Br J Gen Pract. 2001;51(472):924-9.

12. Labelle H, Guibert R, Joncas J, Newman N, Fallaha M, Rivard CH. Lack of scientific evidence for the treatment of lateral epicondylitis of the elbow. An attempted meta-analysis. J Bone Joint Surg Br. 1992;74(5):646-51.

13. Smidt N, Assendelft WJ, Arola H, Malmivaara A, Greens S, Buchbinder R, et al. Effectiveness of physiotherapy for lateral epicondylitis: a systematic review. Ann Med. 2003;35(1):51-62.

14. Newcomer KL, Laskowski ER, Idank DM, McLean TJ, Egan KS. Corticosteroid injection in early treatment of lateral epicondylitis. Clin J Sports Med. 2001;11(4):214-22.

15. Smidt N, van der Windt DA, Assendelft WJ, Devillé WL, Korthals-de Bos IB, Bouter LM. Corticosteroid injections, physiotherapy, or a wait-and-see policy for lateral epicondylitis: a randomised controlled trial. Lancet. 2002;359(9307):657-62.

16. Bisset L, Beller E, Jull G, Brooks P, Darnell R, Vicenzino B. Mobilisation with movement and exercise, corticosteroid injection, or wait and see for tennis elbow: randomised trial. BMJ. 2006;333(7575):939.

17. Struijs PA, Damen PJ, Bakker EW, Blankevoort L, Assendelft WJ, van Dijk CN. Manipulation of the wrist for management of lateral epicondylitis: a randomized pilot study. Phys Ther. 2003;83(7):608-16.

18. Pienimaki TT, Tarvainen TK, Siira PT, Vanharanta H. Progressive Strengthening and stretching exercises and ultrasound for chronic Lateral Epicondylitis. Physiotherapy. 1996;82(9):522-30.

19. Manias P, Stasinopoulos D. A controlled clinical pilot trial to study the effectiveness of ice as a supplement to the exercise programme for the management of lateral elbow tendinopathy. Br J Sports Med. 2006;40(1):81-5. 
20. Tonks JH, Pai SK, Murali SR. Steroid injection therapy is the best conservative treatment for lateral epicondylitis: a prospective randomised controlled trial. Int J Clin Pract. 2007;61(2):240-6.

21. Nagrale AV, Herd CR, Ganvir S, Ramteke G. Cyriax physiotherapy versus phonophoresis with supervised exercise in subjects with lateral epicondylalgia: a randomized clinical trial. J Man Manip Ther. 2009;17(3):171-8.

22. Rompe JD, Decking J, Schoellner C, Theis C. Repetitive low-energy shock wave treatment for chronic lateral epicondylitis in tennis players. Am J Sports Med. 2004;32(3):734-43.

23. Melegati G, Tornese D, Bandi M, Rubini M. Comparison of two ultrasonographic localization techniques for the treatment of lateral epicondylitis with extracorporeal shock wave therapy: a randomized study. Clin Rehabil. 2004;18(4):366-70.

24. Haake M, König IR, Decker T, Riedel C, Buch M, Müller HH, et al. Extracorporeal shock wave therapy in the treatment of lateral epicondylitis : a randomized multicenter trial. J Bone Joint Surg Am. 2002;84-A(11):1982-91.

25. Speed CA, Nichols D, Richards C, Humphreys H, Wies JT, Burnet $\mathrm{S}$, et al. Extracorporeal shock wave therapy for lateral epicondylitis--a double blind randomised controlled trial. J Orthop Res. 2002;20(5): 895-8.

26. Chung B, Wiley JP. Effectiveness of extracorporeal shock wave therapy in the treatment of previously untreated lateral epicondylitis: a randomized controlled trial. Am J Sports Med. 2004;32(7):1660-7.

27. D’Vaz AP, Ostor AJ, Speed CA, Jenner JR, Bradley M, Prevost AT, et al. Pulsed low-intensity ultrasound therapy for chronic lateral epicondylitis: a randomized controlled trial. Rheumatology. 2006;45(5): 566-70.

28. Oken O,KahramanY,AyhanF,CanpolatS, YorganciogluZR, Oken OF. The short-term efficacy of laser, brace, and ultrasound treatment in lateral epicondylitis: a prospective, randomized, controlled trial. J Hand Ther. 2008;21(1):63-7.

29. Runeson L, Haker E. Iontophoresis with cortisone in the treatment of lateral epicondylalgia (tennis elbow) - a double-blind study. Scand J Med Sci Sports. 2002;12(3):136-42.
30. Nirschl RP, Rodin DM, Ochiai DH, Maartmann-Moe C; Group DEXAHES. Iontophoretic administration of dexamethasone sodium phosphate for acute epicondylitis. A randomized, double-blinded, placebo-controlled study. Am J Sports Med. 2003;31(2):189-95.

31. Basford JR, Sheffield CG, Cieslak KR. Laser therapy: a randomized, controlled trial of the effects of low intensity Nd:YAG laser irradiation on lateral epicondylitis. Arch Phys Med Rehab. 2000;81(11):1504-10.

32. Ogueta E. Laser en epicondilitis. Bol Cient Asoc Chil Segur. 2000;2(3):40-5.

33. Lam LK, Cheing GL. Effects of 904-nm low-level laser therapy in the management of lateral epicondylitis: a randomized controlled trial. Photomed Laser Surg. 2007;25(2):65-71.

34. Stergioulas A. Effects of low-level laser and plyometric exercises in the treatment of lateral epicondylitis. Photomed Laser Surg. 2007;25(3):205-13.

35. Garg R,Adamson GJ,DawsonPA, Shankwiler JA,PinkMM. A prospective randomized study comparing a forearm strap brace versus a wrist splint for the treatment of lateral epicondylitis. J Shoulder Elbow Surg. 2010;19(4):508-12.

36. ParkJY, Park HK, Choi JH, Moon ES, Kim BS, Kim WS, etal. Prospective evaluation of the effectiveness of a home-based program of isometric strengthening exercises: 12-month follow-up. Clin Orthop Surg. 2010;2(3):173-8.

37. Başkurt F, Ozcan A, Algun C. Comparison of effects of phonophoresis and iontophoresis of naproxen in the treatment of lateral epicondylitis. Clin Rehabil. 2003;17(1):96-100.

38. Fink M, Wolkenstein E, Karst M, Gehrke A. Acupuncture in chronic epicondylitis: a randomized controlled trial. Rheumatology. 2002;41(2):205-9.

Recebido: 30/09/2011 Received: 09/30/2011

Aprovado: $11 / 03 / 2013$

Approved: 03/11/2013 


\section{ANEXO I - Estratégia de busca utilizada para a base de dados PubMed}

\#1 (Tennis Elbow OR Elbow, Tennis OR Elbows, Tennis OR Tennis Elbows OR Lateral Epicondylitis OR Epicondylitis, Lateral Humeral OR Epicondylitides, Lateral Humeral OR Humeral Epicondylitides, Lateral OR Humeral Epicondylitis, Lateral OR Lateral Humeral Epicondylitides OR Lateral Humeral Epicondylitis OR Bursitis, Radiohumeral OR Epicondylalgia Humeri OR Epicondylitis Humeri OR Epicondylitis Lateralis OR Epicondylitis, External humeral OR Epicondylitis, Radiohumeral OR Epycondylitis Humeri OR External Humeral Epicondylitis OR Humerus Epicondylitis OR Radiohumeral Bursitis OR Radiohumeral Epicondylitis OR Tennis Arm)
\#2 (Physical Therapy Modalities OR Modalities, Physical Therapy OR Modality, Physical Therapy OR Physical Therapy Modality OR Physical Therapy Techniques OR Physical Therapy Technique OR Techniques, Physical Therapy OR Physiotherapy (Techniques) OR Physiotherapies (Techniques) OR Conservative Treatment OR Conservative Therapy OR Nonoperative Treatment OR Nonsurgical Treatment OR Treatment, Conservative)

\#3 (randomized controlled trial [pt]) OR (controlled clinical trial [pt]) OR (randomized [tiab]) OR (placebo [tiab]) OR (randomly [tiab]) OR (trial [tiab]) OR (groups [tiab])) AND (humans [mh])

\#4 (\#1 AND \#2 AND \#3)

\section{ANEXO II - Pontuação dos artigos na escala de qualidade PEDro}

\begin{tabular}{|c|c|c|c|c|c|c|c|c|c|c|c|c|}
\hline Estudo & 1 & 2 & 3 & 4 & 5 & 6 & 7 & 8 & 9 & 10 & 11 & Pontuação \\
\hline Fink, 2002 & Sim & Sim & Não & Sim & Não & Não & Sim & Sim & Não & Sim & Sim & 6 \\
\hline Baskurt, 2003 & Não & Sim & Não & Sim & Não & Não & Não & Não & Não & Sim & Não & 3 \\
\hline Melegati, 2004 & Sim & Sim & Não & Sim & Sim & Não & No & Sim & Não & Sim & Sim & 6 \\
\hline Strujis, 2004 & Sim & Sim & Não & Sim & Não & Não & Sim & Sim & Sim & Sim & Sim & 7 \\
\hline Smidt, 2002 & Sim & Sim & Sim & Sim & Não & Não & Sim & Sim & Sim & Sim & Sim & 8 \\
\hline Chung, 2004 & Sim & Sim & Sim & Sim & Não & Não & Sim & Sim & Sim & Sim & Sim & 8 \\
\hline Speed, 2002 & Sim & Sim & Não & Sim & Sim & Não & Sim & Sim & Sim & Sim & Sim & 8 \\
\hline Haake, 2002 & Sim & Sim & Sim & Não & Sim & Não & Sim & Sim & Sim & Sim & Sim & 8 \\
\hline Runeson, 2002 & Não & Sim & Sim & Não & Sim & Sim & Sim & Não & Não & Sim & Não & 6 \\
\hline Nirschl, 2003 & Sim & Sim & Não & Sim & Sim & Sim & Sim & Sim & Não & Sim & Não & 7 \\
\hline Basford, 2000 & Sim & Sim & Não & Sim & Sim & Sim & Não & Sim & Não & Sim & Sim & 7 \\
\hline Bisset, 2006 & Sim & Sim & Sim & Não & Não & Sim & Sim & Sim & Sim & Sim & Sim & 8 \\
\hline Pienimaki, 1996 & Sim & Sim & Não & Sim & Não & Não & Sim & Sim & Não & Sim & Sim & 6 \\
\hline D'Vaz, 2006 & Não & Sim & Não & Não & Sim & Sim & Sim & Sim & Não & Sim & Sim & 7 \\
\hline Rompe, 2004 & Não & Sim & Sim & Sim & Não & Sim & Sim & Sim & Sim & Sim & Sim & 9 \\
\hline Tonks, 2007 & Sim & Sim & Sim & Não & Não & Não & Não & Não & Não & Sim & Sim & 4 \\
\hline Newcomer, 2001 & Sim & Sim & Não & Sim & Sim & Sim & Não & Sim & Sim & Sim & Sim & 8 \\
\hline Strujis, 2003 & Sim & Sim & Sim & Sim & Não & Não & Sim & Sim & Não & Sim & Sim & 7 \\
\hline Manias, 2006 & Sim & Não & Não & Não & Não & Não & Não & Sim & Não & Sim & Sim & 3 \\
\hline Lam, 2007 & Sim & Sim & Não & Sim & Sim & Não & Sim & Sim & Não & Sim & Sim & 7 \\
\hline Stergioulas, 2007 & Sim & Sim & Sim & Sim & Sim & Não & Sim & Não & Não & Sim & Sim & 7 \\
\hline Oken, 2008 & Sim & Sim & Não & Sim & Não & Não & Sim & Sim & Não & Sim & Sim & 6 \\
\hline Nagrale, 2009 & Sim & Sim & Não & Sim & Não & Não & Sim & Sim & Não & Sim & Sim & 6 \\
\hline Garg, 2010 & Não & Sim & Não & Sim & Não & Não & Não & Não & Não & Sim & Sim & 4 \\
\hline Park, 2010 & Não & Sim & Não & Sim & Não & Não & Não & Não & Não & Sim & Sim & 4 \\
\hline
\end{tabular}

